\title{
Hilbert, Kant y el fundamento de las matemáticas ${ }^{1}$
}

\author{
Carlos Torres Alcaraz
}

U

na de las figuras más sobresalientes de la matemática contemporánea es, sin duda alguna, David Hilbert (1862-1943), no sólo por su destacada obra científica, sino porque en sus manos esta disciplina adquirió una fisonomía que ha perdurado hasta nuestros días, y que se caracteriza por el uso generalizado del método axiomático. Su nombre está indisolublemente ligado a teorías de la más diversa índole, muchas de las cuales le deben en gran medida sus métodos y orientación. ${ }^{2}$ Además de su versatilidad como matemático, podemos decir que su preocupación por tantos y tan diversos temas no se dio en forma aislada, sino que siempre estuvo acompañada por una profunda reflexión en torno a la naturaleza de las matemáticas, donde podemos observar un retorno a la filosofía crítica de Kant, al menos en los siguientes aspectos: 1) niega o evita la metafísica y reduce la filosofía de las matemáticas a una teoría sobre el conocimiento matemático y 2) separa el conocimiento matemático de sus aspectos psicológicos, para sólo concentrarse en su forma lógica. De su concepción de las matemáticas y del vínculo de ésta con la filosofía de Kant es de lo que me quiero ocupar en las siguientes líneas, sobre todo en referencia con la primera fase de sus investigaciones -aquella en que examina los principios de la geometría-, pues en ella se encuentra la raíz de lo que más tarde será un programa tendiente a fundamentar la matemática clásica, que con tanto cuidado afinara en la déca-

${ }^{1}$ Una versión preliminar de este trabajo fue leída en las Jornadas de Historia y Filosofía de la Ciencia que se llevaron a cabo del 20 al 22 de mayo de 1999 en el Centro de Investigación en Matemáticas (CIMAT) en la ciudad de Guanajuato, Gto. Quiero agradecer a Isabel Cabrera, Jaime Óscar Falcón, Juan José Rivaud y Pedro Stepanenko sus finas observaciones.

${ }^{2}$ Entre las áreas en que trabajó se encuentran las siguientes: física matemática, teoría de los invariantes, teoría de los ideales de polinomios, teoría de los números algebraicos, geometría elemental, cálculo de variaciones, teoría de las ecuaciones integrales, análisis funcional, ecuaciones diferenciales y lógica matemática. 
da de los años veintes y que a tantos equívocos ha dado lugar en cuanto a su concepto de esta ciencia. ${ }^{3}$

En efecto, si tomamos como guía la abundante literatura sobre el tema, es muy probable que terminemos por creer que la visión que Hilbert tiene de la matemática es lo que podemos llamar un crudo formalismo, según el cual esta disciplina consistiría en una colección de teorías formales carentes de todo contenido y sujetas a estrictas reglas de manipulación simbólica. Un punto de vista similar al que Carnap sostuviera en la década de los años treintas. ${ }^{4}$ Creo que ésta es una imagen muy limitada de la concepción que Hilbert tiene de las matemáticas y atañe a ciertas ideas propias del último periodo de sus investigaciones en torno al fundamento de las matemáticas. Ciertamente, en ella habla de formalizar las matemáticas a fin de llevar a buen término su fundamentación lógica, mas confundir el método con una posición filosófica me parece un error. Creo, por el contrario, que la propuesta sintáctica propia de esta fase de su carrera opaca la verdadera visión que tenía de las matemáticas y que debemos recuperar. $\mathrm{Al}$ respecto me propongo reconsiderar el papel que asigna al método axiomático y a la formalización en las matemáticas, mostrando con ello que Hilbert, lejos de ese tajante formalismo que se le atribuye, ve en ellas algo más que una colección de teorías formales, y que para él la estricta formalización es tan sólo un instrumento que posibilita el examen de la estructura deductiva de las teorías, a fin de dotarlas de un fundamento lógico. Asimismo, quiero mostrar cómo su concepción de las matemáticas se sirve de algunas ideas extraídas de la filosofía de Kant, lo que permite distinguir su pensamiento del formalismo extremo de Haskel B. Curry o del logicismo de Gottlob Frege y Bertrand Russell. ${ }^{5}$

\section{Los fundamentos de la geometría}

Podemos acercarnos al pensamiento de Hilbert en torno a la naturaleza de las matemáticas observando cómo procede al momento de fundamentar la geometría.

${ }^{3}$ Pese al interés que reviste el llamado programa de Hilbert, en estas líneas no me ocuparé mayormente de él, en parte porque mi propósito es otro, y en parte porque de suyo es digno de un estudio independiente. No obstante, este trabajo se puede entender como un primer paso en esa dirección.

${ }^{4}$ Cf. Rudolf Carnap, The Logical Syntax of Language. Nueva York, Harcourt Brace, 1937.

${ }^{5}$ Cf. Haskell B. Curry, Foundations of Mathematical Logic. Nueva York, Dover, 1976; Gottlob Frege, Grundgesetze der Arithmetik, begriffsschriftlich abgeleitet. Jena, H. Pohle, vol. 1, 1893, y Bertrand Russell, The Principles of Mathematics. Londres, George Allen and Unwin, 1903. 
En 1899 Hilbert publica un libro titulado Grundlagen der Geometrie ${ }^{6}$ (al que nos referiremos como "los Grundlagen") en el que expone una nueva base axiomática para la geometría euclidiana. Se puede decir que en este trabajo se encuentra la primera exposición moderna del método axiomático, en el que las limitaciones de la axiomática material de Euclides se ven por fin superadas. Se trata de la misma noción de teoría axiomática que con tanta naturalidad se ha extendido a prácticamente todas las ramas de la matemática moderna.

En los Grundlagen la geometría es tratada como un sistema hipotéticodeductivo que depende de las definiciones implícitas de los conceptos de los objetos espaciales y de las relaciones que contienen los axiomas, y no de la descripción de su contenido intuitivo. Una novedad que se presenta en el texto es que al sistema de axiomas se le exige que satisfaga los requisitos lógicos de consistencia, independencia y completud, y se incluyen algunas pruebas relativas, construidas a propósito, de tales hechos por medio de ciertas interpretaciones sui generis de los términos primitivos. De este modo, si alguien preguntara en el contexto de la teoría axiomática ¿qué son los puntos?, ¿qué son las líneas?, la única respuesta sería "cualquier sistema de objetos y relaciones entre ellos que satisfaga estos axiomas", y le mostraría la lista de los postulados. Podemos decir entonces que para Hilbert, los axiomas definen de manera implícita los conceptos básicos contenidos en ellos.

Esta postura no fue del agrado de todos, ni fue, como veremos, tan rigurosa como pudiera parecer a primera vista. Por ejemplo, Frege le reprocha que con base en sus definiciones no se puede saber si un reloj de bolsillo es un punto, pues en ningún sitio se da una explicación de este concepto. ${ }^{7}$ Aun así, una respuesta a su pregunta es que un reloj de bolsillo será un punto en caso de que forme parte de un sistema de objetos que satisfagan los axiomas. ${ }^{8}$

Comparativamente, una diferencia radical de los Grundlagen con la exposición axiomática de Euclides es que los objetos denotados por los términos geométricos (puntos, líneas, planos, etcétera) no son entidades intuitivas, sino seres de los que lo único que "sabemos" es lo enunciado por los axiomas, y de los que nada podemos saber que no se deduzca de estos últimos,

${ }^{6}$ David Hilbert, Grundlagen der Geometrie. Leipzig/Berlín, Teubner, 1899. Se cuenta con una traducción al inglés que incorpora algunas adiciones hechas por Hilbert a la edición francesa de 1899: D. Hilbert, Foundations of Geometry. Trad. de E. J. Townsend. La Salle, Open Court Publishing, 1962.

${ }^{7}$ Cf. G. Frege, Philosophical and Mathematical Correspondence. Londres, Blackwell, 1980.

${ }^{8}$ Esta polémica entre Hilbert y Frege es de gran interés y merece algo más que una simple mención como la que aquí hago. No obstante, las limitaciones de espacio me hacen imposible extenderme en ella todo lo que quisiera. 
evitándose de esta manera toda referencia a algo externo al sistema. Asimismo, dado que el propósito de la axiomatización es desarrollar la teoría al margen de la intuición o de la experiencia, los axiomas no se pueden justificar apelando a su evidencia, sino que son simplemente proposiciones que se asumen como punto de partida de la demostración. ${ }^{9}$

En cuanto a su sustento, podemos ver que Hilbert abandona toda pretensión de fundar la geometría en lo real, es decir, de utilizar como soporte la idea de que describe relaciones entre objetos predeterminados. Ya no se tiene, como en Euclides, un conjunto de objetos geométricos privilegiados de cuya realidad se está convencido, ni proposiciones en el sentido clásico del término, que no sólo enuncian un contenido sino que se afirman a título de verdades. Ahora lo único que sabemos de los objetos geométricos es lo que establecen los axiomas, y ya no viene al caso preguntar ¿qué son los puntos y las líneas?, sino ¿qué propiedades tienen los puntos y las líneas? En este sentido, el único fundamento de la teoría axiomática es su coherencia lógica, es decir, la propiedad de ser no contradictoria. ${ }^{10}$

Hilbert aborda este problema en los Grundlagen. De hecho, este ensayo constituye el punto de partida de sus investigaciones en torno al fundamento de las matemáticas, y con base en él pudo implantar el método axiomático no sólo en la geometría, sino en prácticamente todas las ramas de la matemática y la física. El libro contiene un capítulo dedicado a investigar cuestiones que hoy en día se denominan metamatemáticas, en las que los objetos de estudio ya no son aquellos de los que se ocupa la teoría (puntos, líneas, planos, etcétera), sino la teoría misma, sus proposiciones. De estas investigaciones la primordial es la destinada a establecer la consistencia de los axiomas, que sólo logra en forma relativa, pues lo único que consigue es exhibir un

9 Esto último significa que para los fines de la demostración el significado que pudieran tener los axiomas es irrelevante, pues lo único que importa de ellos es el entramado de relaciones a que dan lugar. Por tanto, los términos iniciales, junto con las relaciones básicas, permanecen como algo no definido en el sistema, impidiéndose así que en las demostraciones intervengan elementos extraños a la teoría, como sucede, por ejemplo, en los Elementos de Euclides, donde el significado de los términos iniciales o las figuras que acompañan a la demostración llevan a conclusiones que no se siguen de los axiomas. En este sentido, la obra de Hilbert se debe considerar como una continuación de la de Euclides, en la que los defectos de su construcción habrían de ser corregidos.

${ }^{10}$ En otras palabras, en los Grundlagen lo único que se tiene es un sistema de enunciados formales - axiomas de enlace, de orden, de paralelismo, etcétera- cuya validez no deriva del hecho de que se refieran a objetos claros y distintos, sino de su coherencia lógica. Un movimiento hipotético puro, independiente de la estructura en que se origina, y en el que la verdad de los teoremas está condicionada a la de los axiomas. 
modelo aritmético de los axiomas geométricos, mostrando que no son más que una expresión distinta de los hechos del álgebra lineal y de la teoría de las ecuaciones lineales. ${ }^{11}$

En cuanto al origen de los conceptos y de los axiomas geométricos, Hilbert lo ubica en el ámbito de la intuición, mas no el de la intuición pura, sino en la consideración de lo que la intuición sensible supone. No ve en los axiomas verdades necesarias, sino proposiciones que pueden ser refutadas por la experiencia o incluso ser contradictorias entre sí: la intuición también nos puede engañar (por ello la necesidad de las pruebas de consistencia). En esto no concuerda con Kant, que sostiene que los juicios de la geometría son sintéticos a priori.

\section{El marco teórico de los Grundlagen}

En los Grundlagen podemos reconocer la simiente de las ideas que Hilbert habría de desarrollar más adelante sobre el papel de la axiomatización y su lugar en la matemática moderna. Para él, su obra es una continuación de esa larga tradición que inicia con los griegos, consistente en construir una teoría racional de la estructura del espacio en la que, sin embargo, todos saben de qué es de lo que se está hablando. De ahí que eligiera un conjunto de términos rayanos con la intuición y prefiriera el empleo del lenguaje ordinario antes que el más preciso simbolismo lógico de Peano. ${ }^{12} \mathrm{Al}$ referirse a los axiomas para la geometría dice de éstos que expresan "ciertos hechos, conexos entre sí y fundamentales, de nuestra intuición". ${ }^{13}$ Para aclarar este punto veamos cómo expone uno de sus axiomas:

${ }^{11}$ Sin entrar en detalles diremos que el modelo para la geometría euclidiana se determina con base en el sistema de los números reales, de modo que Hilbert, al probar la consistencia del sistema, supone algo externo a él. No obstante, esto no significa que tal referencia viole el principio de que el desarrollo de la teoría se ha de dar evitando toda referencia a algo externo a la misma. Decir lo contrario es incurrir en un grave error: la prueba de consistencia relativa no establece nada acerca de los objetos geométricos (nada dice de los puntos, las líneas y los planos), sino que informa acerca de las propiedades lógicas de la teoría, lo cual no se contrapone al desarrollo formal de la teoría. En otras palabras: al investigar las propiedades lógicas de la teoría Hilbert recurre a nociones semánticas, pero al derivar sus teoremas no lo hace.

${ }^{12}$ Cf. Selected Works of Giuseppe Peano. Toronto, Universidad de Toronto, 1973, especialmente el trabajo "Studies in Mathematical Logic" de 1897, reproducido en el capítulo XVII.

${ }^{13}$ D. Hilbert, Foundation of Geometry, p. 3. Obviamente, Hilbert se refiere a nuestra intuición geométrica. 
II.4 (Postulado de Pasch) Una línea que corta un lado de un triángulo y que no pasa por ninguno de sus vértices deberá cortar también otro lado del triángulo.

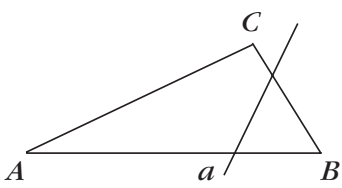

Lo que sorprende de esta presentación es que Hilbert acompaña al axioma con una figura de la que no dice nada y de la que no hace ningún uso más adelante. ¿Por qué entonces su presencia? La respuesta se puede buscar en las palabras introductorias a los Grundlagen. Dice Hilbert:

La geometría -al igual que la aritmética- requiere para su desarrollo sistemático sólo de un reducido número de principios básicos simples. Estos principios son conocidos como axiomas de la geometría. Establecer axiomas para la geometría e investigar la forma en que se relacionan entre sí es un problema que se ha discutido desde la época de Euclides en diversas y admirables contribuciones a la literatura matemática. El problema en cuestión equivale al análisis lógico de nuestra intuición espacial. ${ }^{14}$

La última frase no es circunstancial, sino una declaración de propósitos. Esto lo aclara Hilbert al colocar al principio del libro, a manera de epígrafe, las siguientes palabras, tomadas de la Crítica de la razón pura de Kant: "Así, todo conocimiento humano se inicia con intuiciones, pasa de éstas a los conceptos y termina en las ideas". ${ }^{15}$ Podemos decir entonces que la figura que acompaña

${ }^{14}$ Idem.

15 Immanuel Kant, Crítica de la razón pura. Trad. de Pedro Ribas. Madrid, Alfaguara, 1978, p. 566 [A702, B730]. La voz que Kant utiliza para referirse a la intuición, "Anschauung", tiene en alemán una acepción un tanto más precisa que en español. El significado literal de "Anschauung" sería intuición con una fuerte carga de evidencia. Por ejemplo, Kant diría que un juicio como "el camino más corto entre dos puntos es la línea recta" toma toda su fuerza en la siguiente figura:

y añadiría que sin esta intuición el juicio no tiene fundamento. Es así que el carácter sintético a priori que Kant atribuye a este juicio descansa en la evidencia intuitiva, en el Anschauung. En español, para expresar el origen intuitivo de este juicio, deberíamos 
al postulado de Pasch es la intuición que explica al axioma, y que éste expresa tal hecho de la intuición en términos de una relación entre los conceptos de "línea", "triángulo", "pasar por" y "cortar" (volveremos a esto en la siguiente sección). Pero en su conjunto, la axiomatización constituye una idea en el sentido de Kant, es decir, un objeto de la razón que carece de realidad y que en su perfección sobrepasa la posibilidad de la experiencia.

En efecto, una vez empalmados los conceptos geométricos en una teoría, ésta no es susceptible de una comprobación plena, y veo en ello la razón por la cual Hilbert cita el pasaje de Kant: la geometría, una vez expuesta como un sistema axiomático, tiene la misma condición que las ideas, pues en su conjunto trasciende toda posibilidad de verificación absoluta. Por ejemplo, para verificar el postulado de las paralelas habría que recorrer el espacio al infinito, lo cual resulta imposible. La cita de Kant es por tanto exacta para Hilbert. ${ }^{16}$

En una conferencia pronunciada en 1917 sobre el pensamiento axiomático, Hilbert trata el tema de la axiomatización en su forma general con la siguientes palabras:

Si consideramos en conjunto los hechos que conforman una cierta esfera del conocimiento más o menos comprensiva, nos percataremos de inmediato de que la totalidad de los mismos es susceptible de un orden. La ordenación se lleva a cabo recurriendo a una cierta trama de conceptos relacionados entre sí, de tal manera que a cada objeto y a cada hecho del campo de conocimiento de que se trata le correspon-

darle la siguiente forma: "Veo que el camino más corto...", o "A mi modo de ver, el camino más corto ...", incorporando de esta manera el sentido del vocablo alemán.

${ }^{16}$ Una diferencia entre Hilbert y Kant es que para el segundo los objetos matemáticos son representaciones a priori en la intuición (sin que esto quiera decir que pertenecen sólo a la razón), mientras que para el primero éstos son idealizaciones de lo dado en la intuición sensible (no debemos olvidar que Hilbert ya tiene conocimiento de las geometrías no euclidianas, y no puede seguir argumentando que dichos objetos pertenecen a la intuición pura). En este sentido, Hilbert considera que el espacio matemático es una libre construcción convencional que, aunque creada a partir de intuiciones, en su producto final ya no depende de ellas. Al respecto sostiene que la geometría no es sino parte del sostén teórico de la física, y que su concordancia con el espacio físico se apoya en un ajuste progresivo entre la intuición y la experiencia. No obstante, esto no significa que para él la construcción de una teoría matemática no presuponga cierto tipo de comprensión a priori de la realidad, pero en su opinión este apriorismo no va más allá de la intuición del signo. En este sentido, juzga que Kant sobrestimó el papel y el alcance del a priori en esta disciplina. $C f$. I. Kant, op. cit., pp. 70 y 82-90 [B41 y B59-73] y D. Hilbert, "La connaissance de la nature et la logique", en Einsegnement mathematique, vol. Xxx, 1931, pp. 23-31. 
da, respectivamente, un concepto de esa trama y una relación lógica entre conceptos de la misma. La trama de conceptos no es otra cosa que la teoría de esa esfera del saber. ${ }^{17}$

El análisis lógico al que Hilbert hace referencia en los Grundlagen consiste entonces en precisar los conceptos y la relaciones básicas de la teoría, y en determinar las proposiciones relativas a los elementos que sirven como base de la trama de conceptos, es decir, como punto de partida de la demostración. Es una regla implícita que tras su axiomatización, la teoría se ha de desarrollar al margen de la intuición, las figuras o la experiencia, siendo la demostración el único medio para establecer sus proposiciones. Por tanto, el fruto del análisis lógico de una esfera determinada del conocimiento es una teoría en la que se han reemplazado los objetos propios de ese dominio por conceptos abstractos, los vínculos materiales entre los objetos por relaciones entre los conceptos correspondientes, y la deducción material (es decir, los vínculos causales entre los hechos observables) por deducciones formales que proceden con estricto apego a las leyes de la lógica. Un riguroso orden formal que prescinde del significado de los términos considerados.

\section{Análisis lógico e intuición espacial}

Según parece, Hilbert comparte con Kant la idea de que el conocimiento teórico sólo adquiere significado y objetividad en las intuiciones básicas del entendimiento, y comparte con él la idea de que la intuición es una innegable fuente de conocimientos. ${ }^{18}$ En este sentido, ve en la axiomatización una forma de ordenar las teorías a que da lugar la intuición, esclareciendo sus conceptos y supuestos básicos. Es por ello que la axiomatización de la geometría tiene como punto de partida el análisis lógico de nuestra intuición espacial, a fin de determinar su forma abstracta. Por su parte, los axiomas son el resultado de precisar la forma lógica de ciertos juicios relativos a conceptos espaciales de los que, supuestamente, derivan las propiedades relevantes del espacio. El resultado es una teoría cuya exposición se realiza al margen de toda intuición y que ya no depende de ella. Éste es precisamente el sentido del análisis lógico.

${ }^{17}$ D. Hilbert, "El pensamiento axiomático", en Fundamentos de las matemáticas. Trad. de Luis Felipe Segura. México, UNAM, 1993, p. 23.

${ }^{18}$ Esto no quiere decir que para Hilbert la aritmética transfinita de Cantor o la teoría de los números reales, que suponen el infinito y rebasan el plano de lo intuitivo, no tengan sentido, sino que en realidad no son conocimiento de nada. 
Aclaremos el punto anterior con un ejemplo específico. En parte, el análisis lógico consiste en investigar cómo se vinculan entre sí los puntos y las líneas de nuestra intuición, y considerar el tipo de relaciones a que estos vínculos dan lugar. Por ejemplo, dicho análisis nos lleva a situaciones como la siguiente. Observemos la figura:

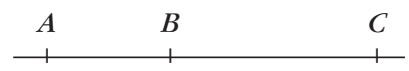

Como se ve, si tres puntos $A, B, C$ están en una línea recta, uno y sólo uno de ellos está entre los otros dos. La validez de esta afirmación descansa en la evidencia de los sentidos, en la intuición espacial, y en ella se observa la manera en que puntos y líneas se enlazan para dar lugar a una relación de orden entre los puntos.

1) La intuición nos "dice" que si nos movemos de $A$ a $C$, pasaremos por $B$.

2) También nos "dice" que si nos movemos de $B$ a $C$ no pasaremos por $A$, incluso si continuamos el movimiento indefinidamente. Esto es parte de la naturaleza de la línea recta según nuestra intuición.

Podemos entonces reunir las proposiciones anteriores y escribir un axioma: II.3 Si $A, B$ y $C$ son tres puntos distintos en una misma línea, entonces uno y sólo uno de ellos está entre los otros dos.

Este último enunciado expresa la forma de nuestra intuición espacial, y cualquier representación de los conceptos de "punto", "línea" y "estar entre" habrá de sujetarse a ella, pues así son los puntos y las líneas en esta geometría. Una característica del axioma II.3 es que garantiza que la línea recta no vuelve sobre sí misma:

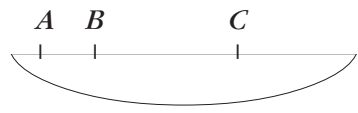

Hasta aquí, Kant estaría de acuerdo con el modo de proceder y las conclusiones alcanzadas. No obstante, para Hilbert el límite que separa el a prio$r i$ de la experiencia se traza de otra manera, reconociendo que la geometría no es una determinación sintética y a priori de las propiedades del espacio, sino una rama del marco conceptual de la física. ${ }^{19} \mathrm{Si}$, por ejemplo, interpretamos "línea" como "rayo de luz" podríamos encontrar, en relación con las figuras anteriores, que al movernos de $B$ hacia $C$, al continuar el movimiento

${ }^{19} C f$. D. Hilbert, "La connaissance de la nature et la logique", en $o p$. cit. 
"en línea recta" también pasemos por $A$ (es decir, que los rayos de luz en el espacio físico siguieran trayectorias cerradas). Pero entonces diríamos que el concepto de "línea recta euclidiana" no es aplicable a los rayos de luz, o que éstos describen trayectorias rectilíneas, pero que el espacio no es euclidiano.

Los físicos relativistas convienen en la segunda alternativa, pero hay que tener claro que se trata de un acuerdo. La matemática no legisla acerca de la estructura del espacio físico, ni dice qué objetos de éste son los correspondientes a la línea recta. Por lo demás no podría hacerlo. El propósito del análisis lógico que practica Hilbert es elaborar una teoría acerca del espacio de nuestra intuición - no acerca del espacio físico o del espacio como condición de posibilidad de toda experiencia-, y ordenar dicha teoría con apego a la lógica. En esto abandona la propuesta de Kant que, por otra parte, ya no era sostenible a principios del siglo veinte. Tampoco es el propósito del análisis lógico dar una definición explícita de los conceptos de "punto", "línea", etcétera, como pretende Frege. ${ }^{20}$

Por otra parte, para llevar a cabo el análisis lógico de nuestra intuición espacial es imprescindible separar la forma del contenido: la lógica no se ocupa de hechos de ninguna especie, sino de relaciones entre conceptos y deducciones. Así, el resultado del análisis no puede ser otra cosa que el establecimiento de un orden de construcción de conceptos y de un orden deductivo entre proposiciones, como habría pretendido Euclides sin lograrlo del todo. ${ }^{21}$

No obstante, y este es uno de los puntos centrales de mi argumento, Hilbert no olvida que no es la lógica sino la intuición la que convierte a la geometría en un desafío para el entendimiento humano, en lugar de uno más entre una infinidad de ejercicios intelectuales arbitrarios. Por ello, el lenguaje de los Grundlagen es, lo repito, el de la geometría elemental, siempre cercano a la intuición. Esto explica la profusión de figuras, algunas de gran belleza, que sin intervenir en las demostraciones aclaran el texto mostrando aquello de lo que se habla. Hilbert practica con excelsitud un juego formal en el que ciertos

\footnotetext{
${ }^{20}$ Por otra parte, nada nos asegura que el análisis lógico será capaz de precisar el contenido de nuestra intuición en todos los casos. Más bien, parece que el lenguaje no es el medio adecuado para hacerlo. El teorema de Lowenheim-Skolem es una evidencia en contra de la capacidad de nuestro lenguaje para abarcar el contenido de nuestra intuición, e incluso la existencia de modelos no estándar numerables de los axiomas de Peano parece ser suficiente.

${ }^{21}$ En este sentido, la obra de Hilbert es efectivamente una continuación y puesta en forma de la de Euclides, quien, como sabemos, no logró independizar la demostración de las intuiciones subyacentes. Prueba de ello es el hecho de que no reconoció ciertos axiomas que correspondían a otras tantas intuiciones $(v . g r$., los axiomas de orden o el postulado de Arquímedes) que, sin embargo, intervienen inadvertidamente en las demostraciones, sobre todo a través de las figuras.
} 
significados prohibidos están siempre ahí, presentes, dando sentido a la teoría, pero sin que se les pueda acusar de nada, pues cuando se les quiere inculpar de algún delito lo único que permanece en su lugar son expresiones vacías. Se trata de un equilibrio que supo mantener a la perfección: separa a las palabras de sus denotaciones, mas no lo suficiente como para que éstas no sigan dando sentido a la teoría. En la geometría el único sustento formal de los teoremas es la demostración, pero es la intuición la que señala el camino.

\section{Consistencia y existencia matemática}

Respecto a la existencia matemática, Hilbert la define por la falta de contradicción, sentido mucho más débil que la existencia empírica. Con ello también evita internarse en el realismo conceptual, conservando a la vez un sentido matemático para los enunciados de esta ciencia. Decir que una noción matemática existe significa simplemente que la podemos caracterizar axiomáticamente sin incurrir en contradicciones. No se trata de que la no contradicción sea la señal de una entidad preexistente, sino que la noción matemática de existencia es lo mismo que ser no contradictorio. Así lo hace saber a Frege en una carta: "De la verdad de los axiomas usted deduce que no pueden contradecirse entre sí, mientras que yo, por mi parte, creo lo contrario, que cuando los axiomas no se contradicen entre sí, por ese motivo son verdaderos, y por ese motivo los objetos que definen existen". ${ }^{22}$

Lejos de tener un carácter absoluto, para Hilbert la verdad y la existencia matemáticas participan de la relatividad de la no contradicción. Por ejemplo, para él la noción central de la teoría de conjuntos de Cantor, la de infinito actual, aunque no se puede representar a priori en la intuición ni corresponde a nada empírico, tendrá plena existencia matemática cuando se pruebe que los axiomas que la definen no se contradicen entre sí. Con ello evita atribuir a la matemática conocimiento de algún tipo de realidad, o al menos convierte esta cuestión en algo irrelevante en relación con sus fundamentos. Esta postura antimetafísica se revela con toda claridad en el siguiente pasaje:

La matemática es una ciencia sin presuposiciones. Para fundamentarla no necesito a Dios, como lo hace Kronecker, o la suposición de una facultad especial de nuestro entendimiento en consonancia con el principio de inducción matemática, como lo hace Poincaré, o la intuición primaria de Brouwer, o, finalmente, como lo hacen Russell y Whitehead,

22 Carta mencionada por I. M. Bochenski, Formale Logik. Friburgo/Munich, K. Alber, 1955, p. 341. 
axiomas de infinitud, reducibilidad o completud que de hecho son suposiciones materiales que no se pueden compensar con pruebas de consistencia. $^{23}$

En contra de lo que pudiera parecer, este pasaje no es un laudo formalista. En él, Hilbert no pretende dar cuenta de la naturaleza o esencia necesaria de las matemáticas, como Haskell B. Curry cuando afirma que "la matemática es la ciencia de los métodos formales", ${ }^{24}$ con lo que proclama que su esencia radica en el método formal como tal. Más bien, lo que dice es que para darle un fundamento, es decir, una base segura a la matemática, no necesita de supuestos metafísicos, sin por ello afirmar o negar que sea obra de Dios, presuponga facultades especiales, o esté referida a un mundo inmaterial de esencias matemáticas. Es más, hacia 1904 Hilbert llega a la conclusión de que en la base de la matemática se halla una forma de pensamiento intuitivo que procede sin supuestos axiomáticos -él le llama matemática finitista- misma que más tarde trataría de convertir en la base para las pruebas de consistencia absoluta.

Aunque no se cuenta con una definición rigurosa de la misma, por matemática finitista se entiende aquella parte de las matemáticas en la que la evidencia descansa en la intuición sensible. En ella, las demostraciones se apoyan en exclusiva en la consideración de objetos concretos (combinaciones de signos, por ejemplo) y sus relaciones mutuas. Esto significa, entre otras cosas, que en la matemática finitista no se acepta ningún tipo de evidencia abstracta como, por ejemplo, la relacionada con las demostraciones de existencia por reducción al absurdo o con la noción de construcción mental. Al contrario, en todas las pruebas consideradas por Hilbert en este dominio sólo se apela a las propiedades combinatorias (espacio-temporales) de las combinaciones de signos consideradas, teniendo como único sostén la intuición del signo (a este punto habremos de volver en la siguiente sección).

Además, Hilbert considera que la matemática clásica es una extensión de esta forma básica del pensamiento intuitivo, la cual se obtiene al adjuntar, a esta última, nociones ideales que nada significan por sí mismas. En esto se arroga las mismas reservas que Kant cuando, en una conocida nota de la Crítica de la razón pura, dice:

El conocimiento de un objeto implica el poder demostrar su posibilidad, sea porque la experiencia testimonie su realidad, sea a priori,

${ }^{23}$ D. Hilbert, "The Foundation of Mathematics", en Jean van Heijenoort, ed., From Frege to Gödel. Trad. de Stefan Bauer-Mengelberg y Dagfinn Føllesdal. Cambridge, Universidad de Harvard, 1967, pp. 464-479.

${ }^{24}$ H. B. Curry, op. cit., p. 14 . 
mediante la razón. Puedo, en cambio, pensar lo que quiera, siempre que no me contradiga, es decir, siempre que mi concepto sea un pensamiento posible, aunque no pueda responder de si, en el conjunto de todas las posibilidades, le corresponde o no un objeto. Para conferir validez objetiva (posibilidad real, pues la anterior es simplemente lógica) a este concepto, se requiere algo más. ${ }^{25}$

En este sentido, y aun a costa de no poder verificar los enunciados de la matemática individualmente, Hilbert se niega a limitar esta ciencia a nociones descriptivas o a la construcción de conceptos en la intuición pura. ${ }^{26} \mathrm{~A}$ diferencia de Brouwer y Poincaré, está dispuesto a admitir cualquier noción por medio de postulados siempre que no se incurra en contradicciones, independientemente de que tales nociones estén o no referidas a intuiciones. ${ }^{27}$ Tal es el caso, por ejemplo, de la noción de infinito actual, en la que ve el complemento ideal de las nociones concretas de la matemática finitista:

El papel que resta al infinito es el de una idea, según la concepción kantiana de ésta, como un concepto de razón que supera toda experiencia y por medio de la cual se complementa lo concreto en el sentido de una totalidad. Pero a la vez, el infinito es una idea en la que podemos confiar sin reservas en el marco de la teoría que acabo de delinear. [La teoría en cuestión es la metamatemática, que aseguraría su consistencia.] ${ }^{28}$

Y si bien la noción de infinito no corresponde a nada en la realidad, Hilbert no ve en ello una razón para dejarla fuera de la matemática: "Podría ocurrir, no obstante, que el lugar propio y justificado del infinito no sea la reali-

${ }^{25}$ I. Kant, op. cit., p. 25 [B XXVII, nota k].

${ }^{26}$ En su trabajo, ya citado, sobre los fundamentos de las matemáticas, dice al respecto: "Convertir en una exigencia universal que cada fórmula individual sea interpretable por sí misma no es de ninguna manera razonable; por el contrario, las teorías son de tal naturaleza que no necesitamos apoyarnos en la intuición o el significado a la mitad de un argumento" ( $C f$. J. van Heijenoort, ed., From Frege to Gödel, p. 475).

${ }^{27}$ A las nociones cuyos objetos no pueden ser dados en ninguna experiencia posible Kant las denomina ideas de razón. Al respecto, admite la posibilidad de extender el conocimiento mediante su adjunción a condición de que el sistema amplificado no sea contradictorio. No obstante, advierte que tal extensión no aumenta el conocimiento teórico y que su función es meramente práctica, punto en el que Hilbert coincide plenamente.

${ }^{28}$ D. Hilbert, "Acerca del infinito", en Fundamentos de las matemáticas, p. 121. 
dad, sino nuestro pensamiento. Y podría muy bien resultar que en éste el infinito asuma una función conceptual absolutamente imprescindible". ${ }^{29}$

\section{La consistencia como único fundamento racional}

Para Hilbert, el único soporte racional (no ontológico) de una teoría es su coherencia lógica, y su única justificación una prueba de consistencia. ${ }^{30}$ Sólo que aquí se presenta un problema: si lo que se pretende es llevar a cabo un análisis de la demostración, no es suficiente con organizar la teoría con apego al método axiomático, sino que es preciso darle una forma concreta, accesible a la intuición que otrora nos dictara los axiomas de la geometría. Como es sabido, en este caso el recurso es la formalización, en la que las proposiciones de la teoría se representan por medio de fórmulas (combinaciones de signos) perfectamente definidas y las formas de argumentación ordinaria se reemplazan por reglas de inferencia explícitas que sólo atañen a la forma de las fórmulas involucradas, no a su contenido o al significado de sus símbolos.

Una representación de este tipo tiene como propósito convertir la demostración en un mosaico de fórmulas expuesto a la mirada, de manera que su correctud se pueda juzgar por medio de su inspección directa. En tal caso el enunciado de consistencia se convierte en un enunciado de la forma "no es posible derivar a partir de estos axiomas y con estas reglas de inferencia dos fórmulas que sean una la negación de la otra" y estaría referido a objetos que caen en el ámbito de la intuición sensible. Una demostración de consistencia de esta clase se podría sustentar en la intuición del signo y caería por tanto en

${ }^{29}$ Ibid., pp. 87-88. En el mismo texto, Hilbert se declara un acérrimo defensor de la matemática transfinita con las siguientes palabras, ahora famosas: "Nadie podrá expulsarnos del paraíso que Cantor ha creado para nosotros", dirigiéndose inequívocamente a Brouwer, quien ha manifestado su rechazo a la noción de infinito actual en matemáticas.

${ }^{30}$ No sólo las teorías matemáticas se hallan en esta situación: junto con ellas también se encuentran muchos otros campos del conocimiento, principalmente los pertenecientes a la física teórica. En cuanto a las pruebas de consistencia, pudiera parecer que, una vez axiomatizada una teoría, bastaría con referirla a otra que frente a ella se localizase en un plano más profundo. Así, por ejemplo, los modelos euclidianos para las geometrías de Riemann y Lobachevsky junto con el modelo algebraico para la geometría euclidiana, muestran que cualquier contradicción que se presente en alguna de ellas conduce necesariamente a un resultado similar en el sistema de los números reales. No obstante, este "reduccionismo" tiene un límite, que al parecer se halla en el terreno de la aritmética de los números enteros y la teoría de conjuntos. Ciertamente, para estas teorías no se tiene ninguna disciplina a la que se pueda apelar, por lo que en su caso el único recurso es probar directamente su consistencia. 
la esfera de la matemática finitista, que si bien no es suficiente para dar cuenta del contenido de las teorías matemáticas, sí lo es, en opinión de Hilbert, para darles un sólido fundamento. Al respecto dice lo siguiente: "El enfoque que consideramos adecuado y necesario para la fundamentación no sólo de las matemáticas puras, sino en general de todo el pensamiento, la comprensión y la comunicación científicas, puede entonces expresarse en una frase diciendo: en un principio era el signo". ${ }^{31}$

Por "signo" Hilbert entiende no los trazos sobre el papel, que serían meras inscripciones que los representan, sino algo "cuya forma es independiente del espacio y del tiempo, así como de las condiciones especiales en las que se produce, de las variaciones insignificantes en su trazado y que, en general, de manera segura puede ser identificado". ${ }^{32}$ Como señala Jean Ladrière, "para Hilbert, la solidez del pensamiento matemático y la validez de sus pretensiones reside finalmente en la intuición del signo, intuición que disfruta de una evidencia privilegiada". ${ }^{33}$ Como ya lo he mencionado, para él esta forma de intuición no sólo se halla en la base de toda consideración de las teorías formales como un factor que nos permite reconocer a priori cierta realidad requerida en su construcción, sino que incluso se encuentra en el punto de partida del conocimiento matemático, permitiéndonos, por ejemplo, reconocer algunas leyes generales de la aritmética cuando se le aplica a un tipo especial de expresiones simbólicas (como, por ejemplo, I, II, I | I, I | | , etcétera), o cuando se examinan ciertas operaciones con ellas (como, por ejemplo, la suma o unión de expresiones: ||$+|||=|||||$, etcétera). ${ }^{34}$ Hilbert difiere en esto de Kant, quien difícilmente admitiría una opción de esta naturaleza, en la que la intuición aplicada a cierta clase de expresiones simbólicas llevaría al conocimiento de proposiciones aritméticas de carácter general. Aun así, Hilbert ve en él un tipo de discernimiento intuitivo en el que el pensamiento básico y general de Kant retiene su importancia, a condición de que el a priori no se considere más que la expresión de ciertas condiciones preliminares e indispensables para el pensamiento y la experiencia. ${ }^{35}$ Como quiera que sea, esta confianza en la intuición del signo ya se encuentra en Kant, quien al referirse al álgebra se expresa con las siguientes palabras:

31 D. Hilbert, "La nueva fundamentación de la matemática”, en Fundamentos de las matemáticas, p. 45.

32 Idem.

33 Jean Ladrière, Limitaciones internas de los formalismos. Trad. de José Blasco. Madrid, Tecnos, 1969, p. 27.

34 Como hemos visto, Hilbert sostiene que la matemática presupone esta forma especial de intuición y que debido a ello no se le puede reducir a la lógica.

35 D. Hilbert, "La connaissance de la nature et la logique", en op. cit., pp. 28-29. 
El mismo procedimiento del álgebra con sus ecuaciones, a partir de las cuales, por reducción, produce la verdad juntamente con su prueba, aunque no es una construcción geométrica, es una construcción característica por la cual se presentan en la intuición los conceptos a través de los signos, especialmente los que se refieren a relaciones de magnitud. Aun sin atender a su elemento heurístico, este método garantiza la ausencia de errores en todas las inferencias por el hecho de poner a la vista cada una de ellas. ${ }^{36}$

Tal parece que Hilbert se inspiró en este pasaje al momento de idear la formalización. Al igual que en el álgebra, en un sistema formal las pruebas son figuras expuestas a la mirada, y la presencia de una contradicción se manifestaría en la existencia de dos pruebas formales que concluirían en fórmulas contradictorias, de la forma $A$ y $\neg A$. De este modo, Hilbert encuentra una manera de transformar el problema de la consistencia en un problema relativo a un juego reglamentado de símbolos, es decir, en un problema de combinatoria.

Sin entrar en detalles sobre cómo se lleva acabo la formalización ni cuáles han sido los resultados obtenidos, podemos decir que para Hilbert la formalización tenía un valor instrumental, no ontológico: el de llevar adelante el examen de la estructura deductiva de las teorías y darles un fundamento lógico.

Esta tarea era especialmente importante en relación con la teoría de conjuntos de Cantor, donde tras la aparición de las paradojas entre 1895 y 1904 se debió rehacer el edificio entero. En su reconstrucción, Hilbert no quiere renunciar a nada: ni a sus elegantes demostraciones -basadas en el principio del tercero excluido-, ni a las nociones transfinitas que hicieron de ella "la flor más admirable que el espíritu matemático ha producido [...] [y] uno de los logros más elevados de la actividad intelectual humana en general" ${ }^{37}$ En esto tampoco se asemeja a un formalista vehemente, sino a un soñador.

\section{La postura antimetafísica}

Debe ahora quedar claro que señalar a Hilbert como el padre de ciertas formas extremas del formalismo es un error histórico que hay que corregir. Me parece que su figura ha sido indebidamente valorada en este terreno, y que el error se debe a que se ha confundido la adopción de un método para resolver

\footnotetext{
${ }^{36}$ I. Kant, op. cit., pp. 587-588 [B762].

${ }^{37}$ D. Hilbert, "Acerca del infinito", en op. cit., p. 90.
} 
un problema específico (el de la consistencia) con el sostén de una postura filosófica. Veamos cómo se expresa Howard Eves:

La tesis formalista es que la matemática se ocupa de sistemas simbólicos formales. De hecho, la matemática se considera como una colección de tales desarrollos abstractos, en el que los términos son meros símbolos y los enunciados son fórmulas en las que figuran estos símbolos; la base última de la matemática no se encuentra en la lógica, sino sólo en una colección prelógica de marcas o símbolos y en un conjunto de operaciones con estas marcas. Como, desde este punto de vista, la matemática carece de un contenido concreto y sólo contiene elementos simbólicos ideales, el establecimiento de la consistencia de las distintas ramas de la matemática se convierte en una parte importante y necesaria del programa formalista. Sin tal prueba de consistencia $a$ látere, todo el estudio no tiene sentido. En la tesis formalista tenemos el desarrollo axiomático de la matemática llevado a su extremo. ${ }^{38}$

No pretendo discutir el alcance de lo dicho en este pasaje. Lo único que me interesa por el momento es la manera en que en él se detalla una corriente de pensamiento en torno a la naturaleza de las matemáticas, para después atribuir a Hilbert su paternidad:

La escuela formalista fue fundada por David Hilbert después de haber concluido su estudio axiomático de la geometría. En su Grundlagen der Geometrie (1899), Hilbert había afinado el método axiomático, llevándolo de la axiomática material de Euclides a la axiomática formal de nuestros días. El punto de vista formalista fue desarrollado más tarde por él para enfrentar la crisis desencadenada por las paradojas de la teoría de los conjuntos y el desafío que significaba la crítica intuicionista para la matemática clásica. ${ }^{39}$

Ciertamente, lo dicho en el último pasaje describe el desarrollo y los planteamientos históricos de Hilbert, mas la caracterización que Eves hace del formalismo en el primer pasaje no corresponde a la concepción de la matemática del mismo Hilbert, que si bien propone formalizar las distintas teorías matemáticas a fin de lograr una prueba absoluta de su consistencia, en

${ }^{38}$ Howard Eves, An Introduction to the History of Mathematics. 4a. ed. Nueva York, Holt Rinehart and Winston, 1976, p. 481.

${ }^{39}$ Idem. 
ningún momento sostiene que ésta sólo sea una colección de teorías formales carentes de contenido. ${ }^{40}$ Por el contrario, para él la formalización es sólo una herramienta, y considera que la matemática sí posee un contenido, si no en un sentido trascendente, al menos en el sentido kantiano de "representaciones a priori", ${ }^{41}$ y ve en la axiomatización un paso inevitable que hay que dar para estructurar racionalmente tal conocimiento. ${ }^{42}$ Lo que sí se encuentra en el pensamiento de Hilbert es la idea de que toda teoría matemática, una vez alcanzada su madurez, se puede reducir a un sistema simbólico abstracto, proceso en el cual el análisis lógico desempeña un papel muy importante.

En cuanto a la naturaleza de los objetos considerados en la matemática, me parece que Hilbert, adoptando una postura kantiana, no puede afirmar, pero tampoco negar, su existencia autónoma, y con base en ello asegurar la consistencia de sus teorías. Por ello es que no puede aducir como prueba de consistencia que los axiomas de la geometría expresen ciertos hechos relativos a nuestra intuición espacial y considerar esto como su fundamento, o aducir que hay una intuición que nos revela las propiedades de los números enteros, y que esto asegura la consistencia de sus axiomas. A sus ojos, el único camino es ofrecer una prueba de su consistencia sin apoyarse en tales suposiciones. ${ }^{43}$

Esto se corrobora al observar que Hilbert siempre evitó participar en los interminables debates en torno a la naturaleza de los objetos matemáticos. Con su silencio pareciera suscribir en el terreno de la filosofía de la matemática las palabras con que Kant inicia el prólogo a la primera edición de la Crítica de la razón pura: "La razón humana tiene el destino singular, en uno de sus campos de conocimiento, de hallarse acosada por cuestiones que no puede rechazar por ser planteadas por la misma naturaleza de la razón, pero a las que tampoco puede responder por sobrepasar todas sus facultades". ${ }^{44}$

En el caso específico de la matemática, tales cuestiones serían las relativas a la existencia de una realidad conceptual o ideal a la cual estarían refe-

${ }^{40}$ De haberse logrado la reducción propuesta por Hilbert, quizá habría habido razones suficientes para sostener esta tesis, y a lo mejor Hilbert habría cambiado su punto de vista al respecto. No obstante, esto nunca lo sabremos.

${ }^{41}$ Véase la Sección primera, Capítulo I de la Doctrina trascendental del método, en la Crítica de la razón pura de Kant.

${ }^{42}$ Algo que Hilbert sí defendería es que el análisis lógico de nuestra intuición espacial puede producir un cuadro completo de la estructura del espacio. Esta es una tesis secundaria de este trabajo.

${ }^{43}$ Aunque no por ello la prueba sería enteramente racional, pues recurriría a la intuición del signo. En realidad Hilbert no suprime el recurso a la intuición: sólo lo desplaza a una zona más segura.

${ }^{44}$ I. Kant, op. cit., p. 7 [AVII]. 
ridas las teorías matemáticas más significativas: la teoría de los números y la teoría de conjuntos. En este sentido, gran parte de la filosofía de las matemáticas la debemos entender como un esfuerzo sostenido por responder a estas cuestiones, sin la esperanza de poder resolverlas en definitiva, y otra parte como el silencio que tal imposibilidad nos impone. Gödel sería un representante de la primera tendencia, y Hilbert un representante de la segunda. Esto implica, entre otras cosas, la necesidad de replantear la visión que se tiene de la posición filosófica de Hilbert. Creo que lo que él hace es llevar la postura kantiana hasta sus últimas consecuencias, guardando un silencio muy próximo al de Wittgenstein en la última frase del Tractatus: "De lo que no se puede hablar, hay que callar", sin que ello signifique que asume la vacuidad de las matemáticas.

\section{Un comentario final}

La tesis de Hilbert, según la cual la consistencia es la única condición que podemos exigir a una teoría para que sea admisible en la matemática, la convierte a ésta en la ciencia de lo posible, donde por posible se entiende aquello que no lleva a contradicción. Con esto queda descartada la idea de que la matemática es el estudio de un orden particular de objetos, y deja al investigador en libertad de desarrollar el tipo de teoría que prefiera, es decir, a no limitarse a nociones referidas a intuiciones o a experiencias. En esto Hilbert ya no concuerda con Kant, aunque hay que tomar en cuenta que entre ellos median más de cien años de historia y la aparición de la matemática moderna, que habría obligado a Kant a modificar su punto de vista (una de cuyas variantes es la de Hilbert). No obstante, esta postura no hace de Hilbert un formalista extremo, pues la caracterización propuesta no tiene en sí ninguna carga ontológica: el investigador está en libertad de atribuir el significado que quiera a sus conceptos; lo único que no se permite es que viole las reglas de la lógica o que se contradiga. Prueba de ello es que las teorías matemáticas que revisten un mayor interés para Hilbert, la aritmética y el análisis, poseen a su juicio un contenido que les es asegurado al margen de la lógica. En todo caso esta tesis tendería, más que a afirmar o negar que las matemáticas poseen algún contenido, a suscribir la máxima cantoriana según la cual la esencia de las matemáticas radica en su libertad. 